EXTENDED REPORT

\title{
Immunolocalisation of opticin in the human eye
}

\author{
S Ramesh, R E Bonshek, P N Bishop
}

Br J Ophthalmol 2004;88:697-702. doi: 10.1136/bjo.2003.031989

\begin{abstract}
Aim: To localise the recently discovered glycoprotein opticin in the adult human eye.
Methods: Polyclonal rabbit antisera were raised against two different opticin peptides. Isolated human vitreous collagen fibrils were extracted with $8 \mathrm{M}$ urea and the extract analysed by SDS-PAGE and western blotting. Paraffin embedded sections from two normal eyes were subjected to immunohistochemical analysis.

Results: Western blot analysis of the vitreous collagen fibril extract specifically identified opticin as a 45$50 \mathrm{kDa}$ component that migrated as a doublet. Opticin was especially immunolocalised to the vitreous humour where labelling was most intense in the basal and cortical vitreous gel and less intense in the central vitreous. In addition, specific staining was observed along the surfaces of adjacent basement membranes including the internal limiting membrane (ILM) and posterior capsule of the lens. In one eye, labelling was also observed on the anterior lens capsule, but no other ocular tissues were specifically labelled. A type XVIII collagen/endostatin antibody labelled several ocular tissues including the ILM and basal vitreous gel.

Conclusion: The immunolocalisation of opticin was confined to the vitreous humour, ILM, and lens capsule. In situ hybridisation studies have previously demonstrated opticin expression by the posterior nonpigmented ciliary epithelium. Thus, the immunolocalisation data support the proposition that the nonpigmented ciliary epithelium secretes opticin into the vitreous cavity where it associates with vitreous collagen and adjacent basement membranes. The staining along the ILM suggests a role for opticin in vitreoretinal adhesion and the co-localisation of opticin with type XVIII collagen/endostatin at the ILM raises the possibility that interactions between these two molecules might contribute to vitreoretinal adhesion.
\end{abstract}

See end of article for authors' affiliations .....................

Correspondence to: P Bishop, Academic Department of Ophthalmology, Royal Eye Hospital, Oxford Road, Manchester M13 9WH UK; paul.bishop@ man.ac.uk Accepted for publication 15 October 2003

$\mathrm{T}$ he vitreous humour is a highly hydrated, transparent, extracellular matrix that is largely bounded by basement membranes comprising the inner limiting lamina (ILL) of the retina and the posterior capsule of the lens. ${ }^{1}$ It is virtually acellular, apart from a few cells (hyalocytes) in the basal and cortical vitreous. The gel-like nature of the vitreous humour is maintained by a dilute meshwork of collagen fibrils. ${ }^{1-3}$ These long, thin fibrils (typically $12-15 \mathrm{~nm}$ in diameter) are composed of three different collagen types (that is, II, IX, and V/XI) ${ }^{1}$ and have a number of proteins closely associated with them, including opticin. ${ }^{4}$

Opticin was originally discovered as a major component within a pool of molecules extracted from the surface of bovine vitreous collagen fibrils. ${ }^{4}$ It is a member of the small leucine rich repeat protein (SLRP) family; these extracellular matrix proteins are characterised by tandem leucine rich repeats flanked by cysteine clusters. ${ }^{5}$ The SLRPs are divided into three classes; opticin belongs to class III along with epiphycan and osteoglycin/mimecan. ${ }^{4}$ While SLRPs are generally proteoglycans, opticin is an exception in that instead of possessing glycosaminoglycan side chain(s), it is a glycoprotein with an amino terminal region containing a cluster of sialylated O-linked oligosaccharides. ${ }^{4}{ }^{6}$ A number of functions have been ascribed to the SLRPs including organisation of the extracellular matrix (by regulating collagen fibril assembly and spacing), and modulation of cell growth and proliferation. ${ }^{5}$ However, the precise functions of opticin are unknown and an important step towards elucidating its role is to determine its tissue distribution.

In the mouse eye, in situ hybridisation studies have demonstrated opticin mRNA expression specifically in the ciliary body region of the optic cup during development and the non-pigmented epithelium (NPE) of the posterior ciliary body of the adult mouse. ${ }^{7}$ Similarly, in situ hybridisation

analyses of an adult human eye demonstrated a strong signal from the posterior NPE. ${ }^{8}$ However, other studies suggest high level expression of opticin in the adult human iris. ${ }^{90}$ Indeed, results from the NEIBank project (neibank.nei.nih.gov/ index.shtml) where cDNA libraries were constructed from different adult human ocular tissues and clones randomly sequenced showed remarkably that opticin was the sixth commonest clone in the iris library, seventh commonest clone in the optic nerve library, and 27th commonest clone in the retinal pigment epithelium library. Furthermore, Friedman et $a l^{11}$ have immunolocalised opticin to a number of human ocular tissues including the cornea, iris, ciliary body, vitreous, choroid, and retina using an antibody generated against an amino terminal peptide of opticin. In view of our inability to reconcile these various observations we have re-evaluated the immunolocalisation of opticin in the adult human eye.

\section{MATERIALS AND METHODS}

\section{Antisera}

Two polyclonal rabbit antisera were raised against opticin peptides. The OPT-A antiserum was raised against a synthetic peptide (VLNPDNYGEVIDLSNYEELTDYGDQLPEVK) corresponding to a sequence in the amino terminal region of human opticin, whereas the OPT-C antiserum was raised against a synthetic peptide (LPRLPIGRFT) corresponding to the sequence of the carboxy terminus of human opticin. A polyclonal antibody raised against type XVIII collagen/ endostatin (Ab-1) was obtained from the Lab Vision Corporation (Fremont, USA).

Abbreviations: ILL, inner limiting lamina; ILM, internal limiting membrane; NPE, non-pigmented epithelium; SLRP, small leucine rich repeat protein 


\section{Extraction of opticin and western blotting}

Vitreous humour was obtained from the eye of a 26 year old donor after removal of the corneoscleral rim for transplantation, and was stored at $-70^{\circ} \mathrm{C}$. The tissue had been obtained from the Manchester Royal Eye Hospital Eye Bank and permission had been given for use of the ocular tissue in research. The vitreous sample was thawed and centrifuged to obtain a collagenous pellet. The pellet was washed with TRIS buffered saline containing $0.15 \mathrm{M} \mathrm{NaCl}, \mathrm{pH} 7.6$ (TBS) and subsequently with $1 \mathrm{M} \mathrm{NaCl}$ in TRIS buffer. The pellet was then extracted with $8 \mathrm{M}$ urea, and the extract subjected to SDS-polyacrylamide gel electrophoresis (PAGE) using a 4$12 \%$ Bis-TRIS gradient gel with MOPS buffer (Invitrogen) according to the manufacturer's instructions. A goat antirabbit horseradish peroxidase secondary antibody (Sigma) diluted 1:1000 in TBS was used. Western blotting and immunodetection were performed with the enhanced chemilumiscent system as described previously. ${ }^{4}$

\section{Immunohistochemistry}

Paraffin embedded sections of two normal eyes (archived tissue following exenterations) from a 45 year old woman and a 71 year old man were used for the immunohistochemical analyses. The slides were dewaxed and hydrated through graded alcohols to water. The sections were then placed in TBS for 5 minutes before blocking for 20 minutes with horse serum diluted 1:5 in TBS. For the immunolocalisation of opticin, sections were incubated for 1 hour with either the OPT-A antiserum diluted (in TBS) between 1:2000 and $1: 10000$ or the OPT-C antiserum diluted between $1: 100$ and 1:1000. Endogenous peroxidase activity was then blocked by placing the sections in peroxidase block solution (Dako Laboratories) for 5 minutes. The Vectastatin Elite Universal ABC kit (Vector Laboratories, Burlingame, CA, USA) was used for detection of the primary antisera. The slides were incubated for 30 minutes with a biotinylated universal horse anti-mouse/rabbit IgG secondary antibody (an antibody from Vector Laboratories that recognises rabbit and horse $\operatorname{IgG}$ equally well) diluted 1:50 in TBS, before washing with TBS and then placing in TRIS buffer for 5 minutes. The Vector $\mathrm{ABC}$ Reagent was applied for 30 minutes at room temperature. The slides were then washed with TBS and exposed to the Vector VIP staining agent for 3-5 minutes, which produced a magenta colour. The sections were counterstained with haematoxylin, dehydrated, cleared, and mounted. Controls included the use of pre-immune serum instead of primary antibody and experiments using a blocking peptide (that is, the peptide used to generate the antiserum) mixed with the antiserum in TBS at a final concentration of $5 \mu \mathrm{g} / \mathrm{ml}$. The protocol used for type XVIII collagen/endostatin immunolocalisation was similar except that the sections were digested with trypsin $(0.1 \% \mathrm{w} / \mathrm{w}$ in TRIS buffer containing $7 \mathrm{mM} \mathrm{CaCl}_{2}$ ) for 12 minutes at $37^{\circ} \mathrm{C}$ after the hydration step, and were incubated with the Ab-1 antibody (diluted 1:100) overnight.

\section{RESULTS}

\section{Western blotting}

The extract from the collagen fibrils was subjected to western blotting with the OPT-A antiserum revealing a 45-50 kDa component (fig 1). Similar results were obtained with the OPT-C antiserum (data not shown). These results are consistent with published data showing that tissue extracted and recombinant bovine opticin are labelled with the OPT-A and OPT-C antisera and migrate as a diffuse 45-52 kDa component during SDS-PAGE. ${ }^{4}$ The human opticin migrated as a doublet; similarly the $45-52 \mathrm{kDa}$ component produced by SDS-PAGE of bovine opticin sometimes migrates as a discernible doublet. ${ }^{4}{ }^{6}$

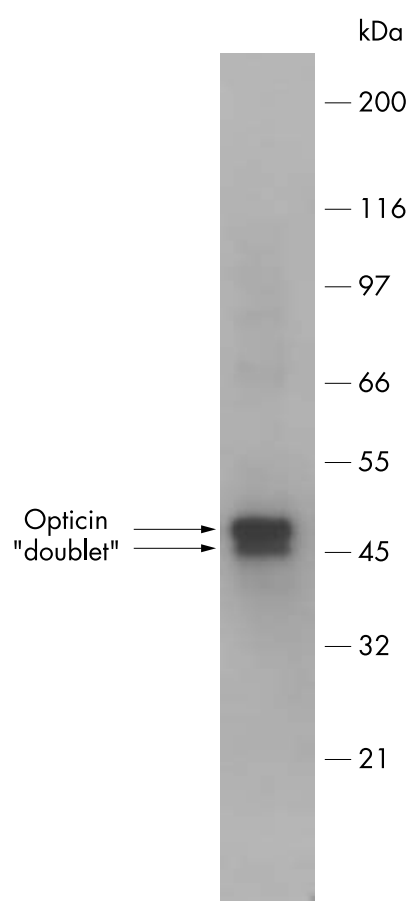

Figure 1 Western blot with the OPT-A antiserum (1:10000 dilution) of an $8 \mathrm{M}$ urea extract from human vitreous collagen fibrils.

\section{Immunolocalisation}

Opticin immunoreactivity was seen almost exclusively in the vitreous and its immediate boundaries. The OPT-A and OPT$\mathrm{C}$ antisera produced similar patterns of labelling in both of the eyes (fig 2A and B). In some sections, there was weak patchy staining of the iris, ciliary body, sclera, and some retinal blood vessels at higher concentrations of the primary antisera. However, on using the pre-immune sera at the same concentration, there was similar staining of these tissues (fig 2C). The pre-immune serum did not produce any significant labelling of the vitreous (fig $2 \mathrm{C}$ ). When the OPTA antiserum was used with the blocking peptide, there was very weak, scattered non-specific labelling of the sections and the vitreous labelling was almost completely abolished (fig 2D).

The most intense labelling was observed in the basal vitreous (figs 2 and 3), especially that close to the surface of the NPE and the retina. The opticin labelling at the ora serrata highlighted (in both eyes) a tongue-like extension of retinal tissue overhanging the most posterior NPE (fig 3A and $\mathrm{B})$. Because the ILL is very thin in the anterior retina it was impossible to determine, at a light microscopic level, whether there was opticin labelling on the retinal aspect of this basement membrane. Focal incursions of opticin labelling into the NPE monolayer were observed (fig 3B). In addition, there were patches of opticin labelling associated with the surface of NPE in the posterior pars plicata (fig 3C).

The labelling became progressively weaker from the cortical vitreous towards the centre of the vitreous cavity (fig 4A); immunolabelling was most intense along the internal limiting membrane (ILM) (fig 4B and C). Some peg-like extensions of the vitreous into the innermost retina were observed but it is unclear whether the staining extended through the ILL or was contained within infoldings of the ILL (fig 4B and C).

There was strong labelling of the anterior hyaloid membrane, especially where it was adherent to the posterior surface of the lens (fig 5A). In the sections from the 45 year old woman, there was also labelling associated with the anterior (fig 5B) and inferior (not shown) surfaces of the lens capsule, although this was not obvious in the eye from the 


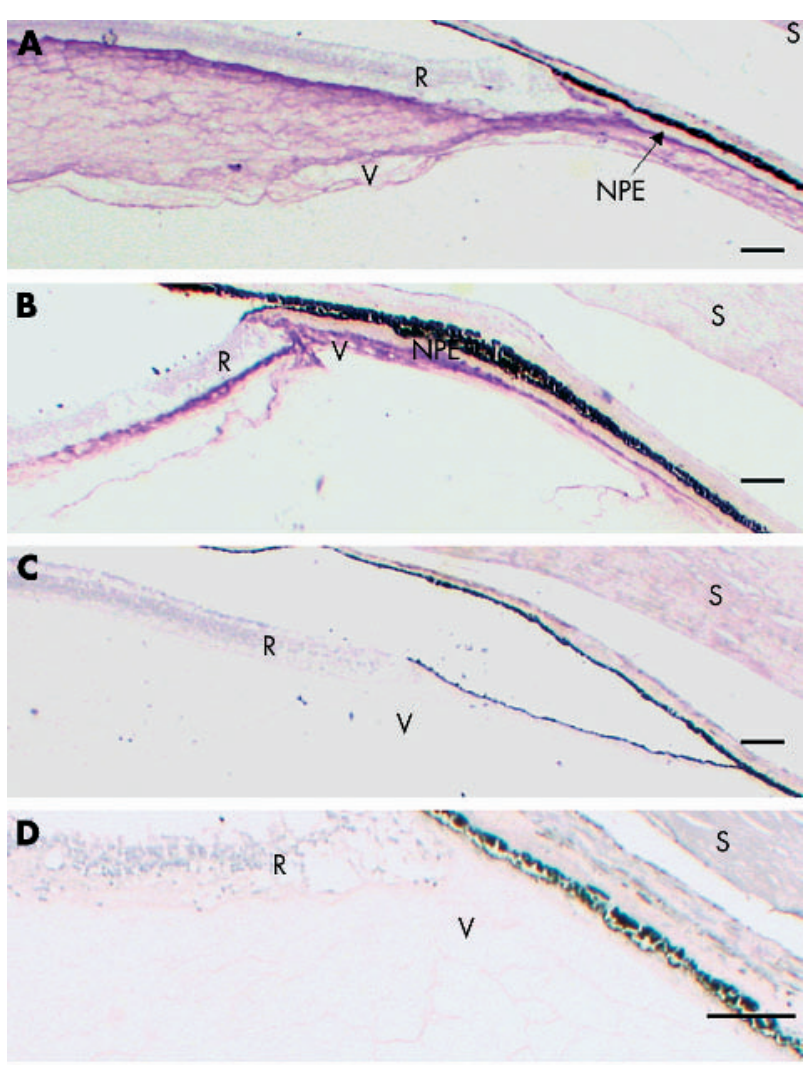

Figure 2 Immunolocalisation of opticin (magenta stain) in the region of the anterior vitreous base using OPT-A antiserum (A) and OPT-C antiserum (B). Controls included OPT-A pre-immune serum (C) and OPTA antiserum with blocking peptide (D). Scale bar $10 \mu \mathrm{m}$. $R=$ retina, $\mathrm{NPE}=$ non-pigmented epithelium, $\mathrm{V}=$ vitreous, $\mathrm{S}=$ sclera.

71 year old subject. The labelling associated with the anterior surface of the lens was wispy, thin and discontinuous (fig 5B). There was intense labelling of the anterior hyaloid membrane as it bridged between the lens and ciliary body (fig 5C). Close inspection revealed that this intense staining was associated with thin, regular, linear stain-excluding bands within the anterior hyaloid membrane that appeared to attach to, or be continuous with, the adjacent lens capsule and the basement membrane of the NPE (fig 5D).

The immunolocalisation of opticin along the ILM suggested that it might contribute to vitreoretinal adhesion and as type XVIII collagen may also contribute to this adhesion (see discussion), the labelling of the opticin antisera and that of a type XVIII collagen/endostatin antibody (Ab-1) were compared. Although the labelling produced by the Ab-l antibody was generally weak, it did produce a very similar pattern to the opticin labelling by being relatively intense at the ILM and revealing peg-like extensions of the vitreous into the innermost retina (fig 6A). Type XVIII collagen/endostatin labelling was also seen in the basal vitreous (fig 6B) and, unlike for opticin, was observed in other ocular tissues including the ciliary body, iris and trabecular meshwork (data not shown).

\section{DISCUSSION}

In this study, we have shown specific labelling with opticin antisera of the vitreous gel, and associated with the innermost ILM and the surface of the lens capsule. In order to validate our results we used two different antisera and confirmed their specificity by western blotting, and included a number of controls including using pre-immune sera and employing peptide blocking experiments.

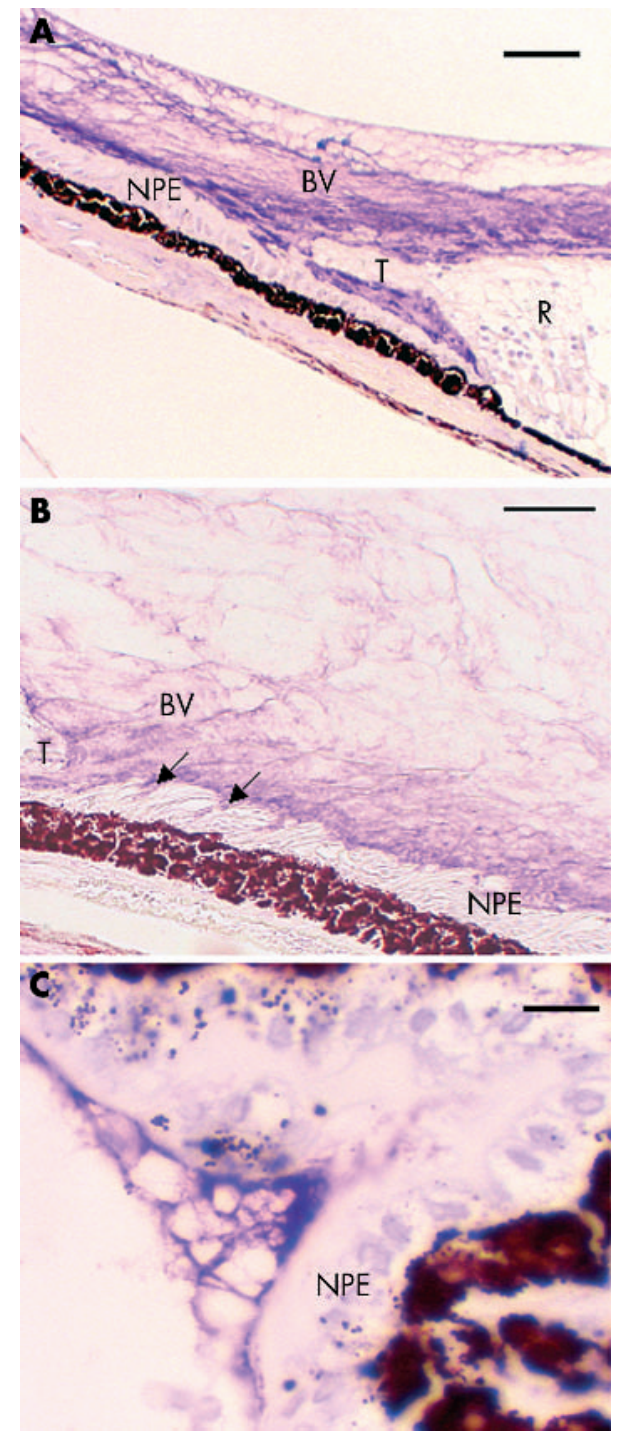

Figure 3 Immunolocalisation of opticin at the vitreous base using OPTA antiserum. (A) Ora serrata of the 71 year old eye; scale bar $10 \mu \mathrm{m}$. (B) Pars plana of the 45 year old eye showing incursions of labelling into the NPE monolayer (arrows); scale bar $5 \mu \mathrm{m}$. (C) A focal patch of labelling on the surface of the NPE in the posterior pars plicata of the 71 year old eye; scale bar $2 \mu \mathrm{m}$. R= retina, NPE = non-pigmented epithelium, $\mathrm{BV}=$ basal vitreous, $\mathrm{T}=$ tongue-like anterior extension of retina at the ora serrata.

The western blotting experiments with our antisera revealed a specific $45-50 \mathrm{kDa}$ component in the human vitreous collagen extract that was similar in all respects to the opticin component from bovine vitreous. ${ }^{4}{ }^{6}$ Friedman et al ${ }^{11}$ have published an immunolocalisation study showing a much wider distribution of opticin labelling including the iris, retina, cornea, choroid, ciliary body, and the vitreous. However, the antibody used by Friedman et al primarily recognised a larger $62 \mathrm{kDa}$ component in ocular tissue extracts following SDS-PAGE and western blotting that they identified as opticin, while also labelling weaker bands at $45 \mathrm{kDa}$ and $38 \mathrm{kDa} .{ }^{11}$ We suggest that their antibody may have cross reacted with other ocular components to produce non-specific immunolocalisation.

It is difficult to reconcile our results with those from the NEIBank that suggest high levels of opticin expression by the optic nerve, iris, and RPE. While it is possible that cells in the optic nerve head could secrete opticin into the vitreous cavity or that opticin is present in the retrobulbar optic nerve 

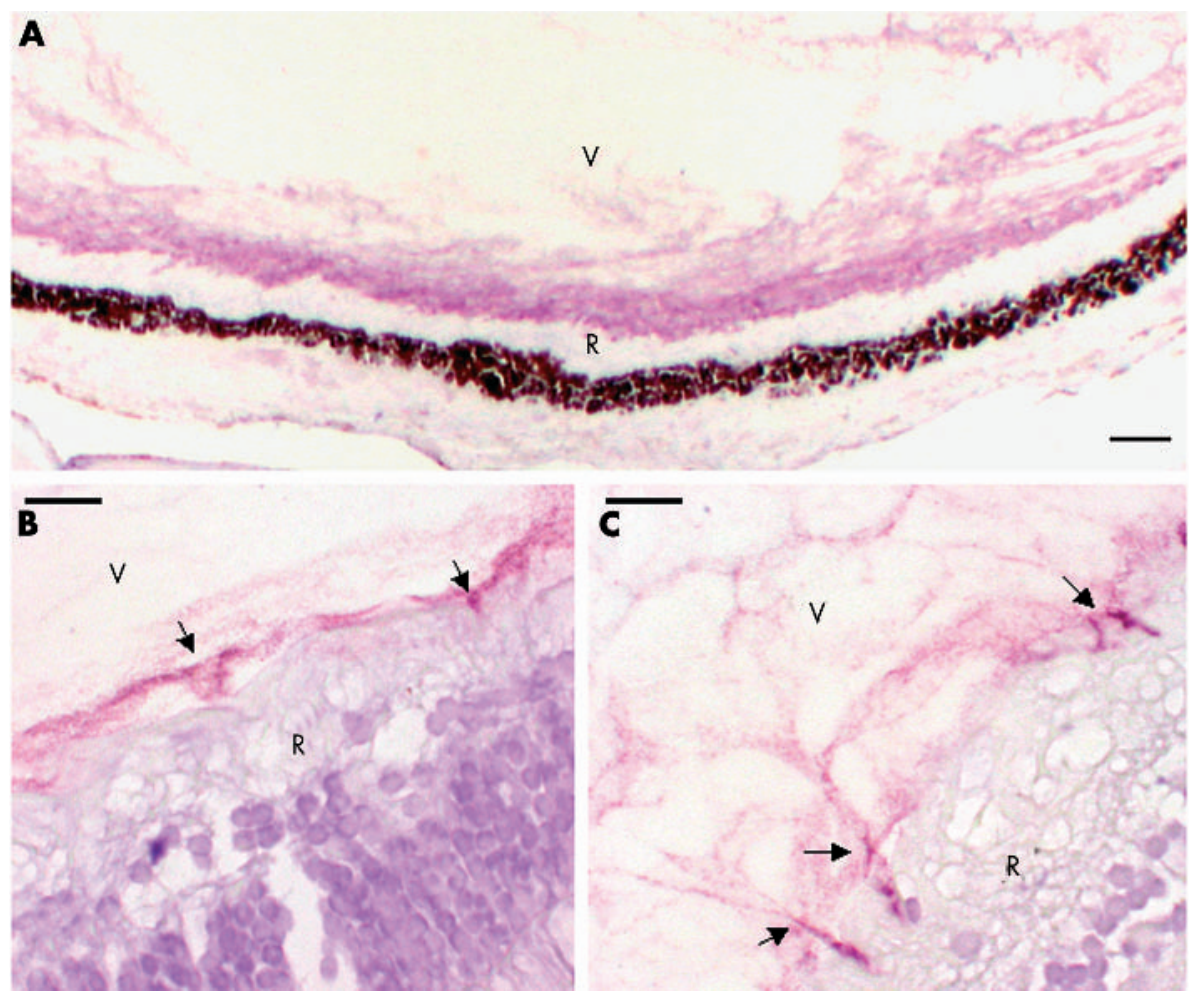

Figure 4 Immunolocalisation of opticin in the post-equatorial vitreous of 45 year old eye using OPT-A antiserum. (A) Overview; scale bar $10 \mu \mathrm{m}$. (B and C) Vitreoretinal interface showing intense labelling of the innermost ILM and peg-like extensions (arrows); scale bar $2 \mu \mathrm{m}$. $\mathrm{R}=$ retina, $\mathrm{V}=$ vitreous
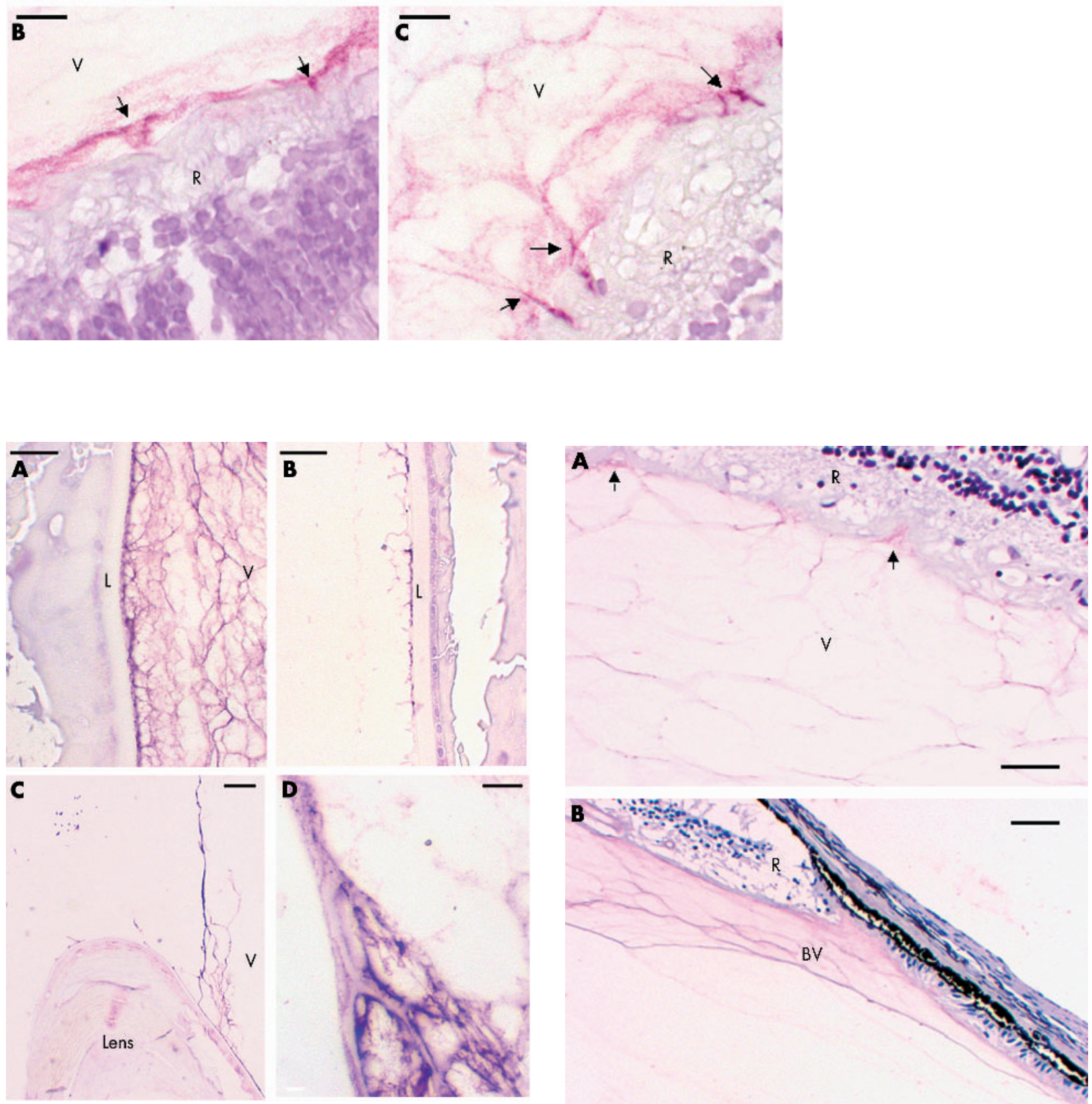

Figure 5 Immunolocalisation of opticin in the anterior hyaloid membrane of 45 year old donor eye. (A) Posterior surface of lens using OPT-A antiserum; scale bar $5 \mu \mathrm{m}$. (B) Anterior surface of lens from 45 year old donor using OPT-A antiserum; scale bar $5 \mu \mathrm{m}$. (C) Lens and anterior hyaloid face using OPT-C antiserum; scale bar $10 \mu \mathrm{m}$. (D) Anterior hyaloid face using OPT-A antiserum; scale bar $2 \mu \mathrm{m}$. L=lens capsule, $V=$ vitreous.

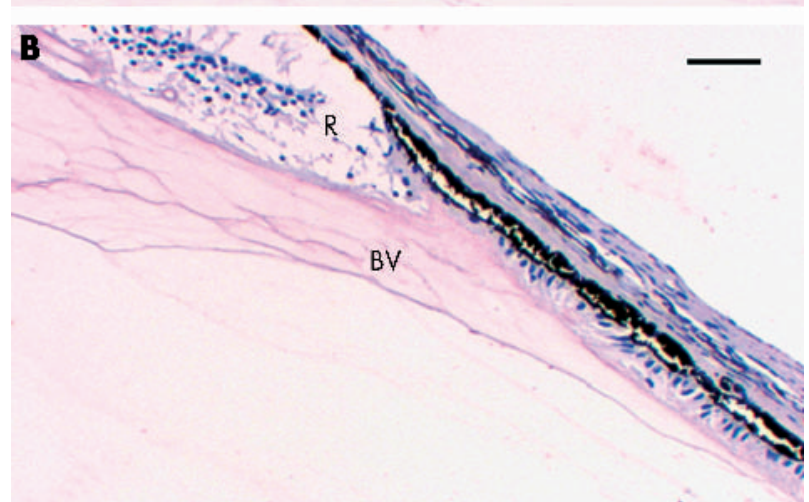

Figure 6 Immunolocalisation of type XVIII collagen/endostatin in the 45 year old donor eye. (A) Vitreoretinal interface showing labelling of the ILM and peg-like extensions (arrows); scale bar $5 \mu \mathrm{m}$. (B) The vitreous base region; scale bar $10 \mu \mathrm{m}$. $R=$ retina, $V=$ vitreous, $\mathrm{BV}=$ basal vitreous 
(which was not visualised in this study), it is difficult to visualise how opticin secreted by the iris cells or the RPE could mainly be destined for the vitreous. The explanation for the discrepancy may lie in the possibility that tissue from the ciliary body was inadvertently included with the iris and RPE tissue used to generate the cDNA libraries in the NEIBank project.

The highly specific and characteristic pattern of labelling of opticin in the posterior segment complements our earlier in situ hybridisation studies on mouse and human eyes, where opticin expression was localised to the posterior part of the ciliary $\mathrm{NPE}^{7}$ - that is, the pars plana and the posterior pars plicata. The NPE would thus be predicted to secrete opticin into the vitreous cavity and, indeed, that is where most of the staining was localised in this study. In the vitreous, opticin appeared to co-localise with collagen as the strongest labelling was found in the areas that contain the highest concentrations of collagen fibrils-that is, the basal and cortical vitreous gel. This might have been predicted as opticin was originally discovered associated with the surface of vitreous collagen fibrils. ${ }^{4}$

The basal vitreous is an annular zone straddling the ora serrata and extending $1.5-2 \mathrm{~mm}$ into the pars plana and up to $3 \mathrm{~mm}$ posterior to the ora serrata. ${ }^{12}$ We have recently published evidence that the basal retina synthesises vitreous collagen and this collagen then breaks through the thin basal ILL and intertwines with the cortical vitreous collagen to form the posterior vitreous base. ${ }^{12}$ However, we could not determine whether the collagen on the retinal side of the ILL labelled with opticin as the ILL in this region is too thin to be visualised by light microscopy.

The ILL is a basement membrane which, on its retinal aspect, adheres to the Müller cell footplates and, on the other side, is attached to the cortical vitreous and these three structures constitute the ILM. ${ }^{13}$ The collagen fibrils of the vitreous cortex do not, in general, insert directly into the ILL but are oriented parallel to the retinal surface and attachment between the vitreous collagen fibrils and ILL may be maintained by intermediary molecules. ${ }^{1}$ An additional form of adhesion at the vitreoretinal interface may be provided by the peg-like structures in the post-basal vitreoretinal interface (fig 4C and D; fig 6A). It is not clear from this study whether these peg-like structures penetrate the ILL or just indent the ILL on the surface of the retina; it is likely that electron microscopy would be required to resolve this issue.

Opticin labelling was closely associated with the ILM. In addition, it was found associated with the lens capsule, which is a basement membrane. These data suggest that opticin may bind to basement membranes. In one of the two eyes, opticin labelling was observed on the inferior and anterior lens capsule. It is possible that opticin secreted by the NPE of the pars plicata (fig 3C) could localise to the inferior and anterior capsule. Another possibility is that this eye was abnormal and had, for example, pseudoexfoliative material on the anterior capsule that contained opticin.

The basement membrane components that have been identified in the ILL include type IV collagen, type XVIII collagen, laminin, nidogen-1, agrin, and perlecan. ${ }^{14}$ Type IV collagen forms a scaffold-like network that stabilises the basement membrane structure while laminin molecules form an independent scaffold as well as interacting with receptors in the cell membrane. ${ }^{15-17}$ Type XVIII collagen has been identified in the chick vitreous and chick and mouse ILM, ${ }^{14}{ }^{18}{ }^{19}$ and evidence of vitreoretinal separation was found in type XVIII collagen null mice indicating a role for this collagen in vitreoretinal adhesion. ${ }^{19}$ Here we used an antibody (Ab-1) that recognises a soluble, anti-angiogenic fragment of type XVIII collagen called endostatin, so this antibody recognises both intact type XVIII collagen and the endostatin fragment. Our results demonstrated the colocalisation of opticin and type XVIII collagen/endostatin at the ILM suggesting that both of these molecules might be important in vitreoretinal adhesion. Rhodes, ${ }^{20}$ in an ultrastructural study of the mouse vitreoretinal interface, described a "fine filamentous network" of glycoprotein associated with the cortical vitreous collagen fibrils and the ILL and suggested that this might be important in vitreoretinal adhesion; it is possible that the glycoprotein identified in his study was opticin. Taken together these suggest the possibility that opticin acts as an intermediary in the adhesion of vitreous collagen fibrils to the ILL, possibly by interacting with type XVIII collagen.

It is known that concurrent with the process of age related vitreous liquefaction, there is also weakening of the adhesion between the cortical vitreous gel and the ILL. These processes eventually result in posterior vitreous detachment in about $25 \%$ of the population. ${ }^{1}$ While the importance of persistent vitreoretinal adhesion following incomplete posterior vitreous detachment is recognised in the pathogenesis of rhegmatogenous retinal detachment, macular hole formation, and some forms of cystoid macular oedema, the molecular mechanisms of vitreoretinal adhesion are poorly understood. Age dependent changes in the molecules on the surface of the vitreous collagen fibrils (including opticin) may predispose the gel to liquefaction and/or weakening of the vitreoretinal adhesion. Perhaps opticin acts as "biological glue" between the vitreous collagen fibrils and the ILL and therefore may provide a therapeutic target for the pharmacological induction of posterior vitreous detachment.

\section{ACKNOWLEDGEMENTS}

The authors gratefully acknowledge the assistance provided by the Histology Laboratory at Manchester Royal Infirmary, UK and thank Professor David McLeod for critically reading the manuscript. PNB is a Wellcome Trust Senior Research Fellow in Clinical Science.

\section{Authors' affiliations}

S Ramesh, R E Bonshek, P N Bishop, Research Group in Eye and Vision Sciences, The Medical School, University of Manchester, UK

P N Bishop, Wellcome Trust Centre for Cell-Matrix Research, School of Biological Sciences, University of Manchester, UK

\section{REFERENCES}

1 Bishop PN. Structural macromolecules and supramolecular organization of the vitreous gel. Prog Ret Eye Res 2000;19:323-44.

2 Bos KJ, Holmes DF, Meadows RS, et al. Collagen fibril organisation in mammalian vitreous by freeze etch/rotary shadowing electron microscopy. Micron 2001;32:301-6.

3 Mayne R, Brewton RG, Ren ZX. The vitreous body and zonular apparatus. In: Harding JJ, ed. Biochemistry of the eye. Chapman and Hall: London, 1997:135-43.

4 Reardon AJ, Le Goff M, Briggs MD, et al. Identification in vitreous and molecular cloning of opticin, a novel member family of leucine-rich repeat proteins of the extracellular matrix. J Biol Chem 2000;275:2123-9.

5 lozzo RV. The biology of the small leucine rich proteins - functional network of interactive proteins. J Biol Chem 1999;274:18843-6.

6 Le Goff MM, Hindson VJ, Jowitt TA, et al. Characterisation of opticin and evidence of stable dimerisation in solution. J Biol Chem 2003;278:45280-7.

7 Takanosu M, Boyd TC, Le Goff M, et al. Structure, chromosomal location and tissue-specific expression of the mouse opticin gene. Invest Ophthalmol Vis Sci 2001:42:2202-10.

8 Bishop PN, Takanosu M, Le Goff M, et al. The role of the posterior ciliary body in the biosynthesis of vitreous humour. Eye 2002;16:454-60.

9 Friedman JS, Ducharme R, Raymond V, et al. Isolation of a novel iris-specific and leucine-rich repeat protein (oculoglycan) using differential selection. Invest Ophthalmol Vis Sci 2000;41:2059-66.

10 Hobby P, Wyatt K, Gan W, et al. Cloning, modeling, and chromosomal localization for small leucine-rich repeat proteoglycan (SLRP) family member expressed in human eye. Mol Vis 2000;6:72-8.

11 Friedman JS, Faucher $M$, Hiscott $P$, et al. Protein localization in the human eye and genetic screen of opticin. Hum Mol Genet 2002;11:1333-42.

12 Wang J, Mcleod D, Henson DB, et al. Age-dependent changes in basal retinovitreous adhesion. Invest Ophthalmol Vis Sci 2003;44:1793-800.

13 Foos RY. Vitreoretinal juncture; topographical variations. Invest Ophthalmol 1972;11:801-8. 
14 Halfter W, Dong S, Schurer B, et al. Composition, synthesis, and assembly the embryonic chick retinal basal lamina. Dev Biol 2000;220:111-28.

15 Mayne R. The eye. In: Royce P, Steinmann B, eds. Connective tissue and its heritable disorders. New York: Wiley-Liss, 2001:131-41.

16 Timpl R. Macromolecular organization of basement membranes. Curr Opin Cell Biol 1996;8:618-24.

17 Colognato H, Yurchenco PD. Form and function: the laminin family of heterotrimers. Dev Dyn 2000;218:213-34.
18 Halfter W, Dong S, Schurer B, et al Collagen XVIII is a basement membrane heparan sulphate proteoglycan, J Biol Chem 1998;273:25404-12.

19 Fukai N, Eklund L, Marneros AG, et al. Lack of collagen XVIII/endostatin results in eye abnormalities. EMBO J 2002:21:1534-44.

20 Rhodes RH. An ultrastructural study of the complex carbohydrates of the mouse posterior vitreoretinal juncture. Invest Ophthalmol Vis $\mathrm{Sci}$ 1982;22:460-77. 